\title{
A self-consistent microscopic model of Coulomb interaction in a bilayer system as an origin of Drag Effect Phenomenon
}

\author{
K. Güven ${ }^{\mathrm{a}, \mathrm{b}}$, A. Siddiki ${ }^{\mathrm{c}, *}$, P.M. Krishna ${ }^{\mathrm{a}}$, T. Hakioğlu ${ }^{\mathrm{a}, \mathrm{d}}$ \\ ${ }^{a}$ Physics Department, Bilkent University, Ankara 06800 Turkey \\ ${ }^{\mathrm{b}}$ Nanotechnology Research Center, Bilkent University, Ankara 06800 Turkey \\ ${ }^{\mathrm{c}}$ Ludwigs-Maximillians University, Physics Department, LS von Delft, D-80333 Munich, Germany \\ ${ }^{\mathrm{d}}$ UNAM Material Science and Nanotechnology Research Institute, Bilkent University, Ankara 06800, Turkey
}

Available online 29 September 2007

\begin{abstract}
In this work we implement the self-consistent Thomas-Fermi model that also incorporates a local conductivity model to an electron-electron bilayer system, in order to describe novel magneto-transport properties such as the Drag Phenomenon. The model can successfully account for the poor screening of the potential within the incompressible strips and its impact on the inter-layer Coulomb interaction. An externally applied current in the active layer results in the tilting of the Landau levels and built-up of a Hall potential across the layer, which, in turn, induces a tilted potential profile in the passive layer as well. We investigate the effect of the current intensity, temperature, magnetic field, and unequal density of layers on the self-consistent density and potential profiles of the bilayer system.
\end{abstract}

(C) 2007 Elsevier B.V. All rights reserved.

PACS: 73.20.Dx; 73.40.Hm; 73.50.-h; 73.61-r

Keywords: Edge states; Quantum Hall effect; Screening; Quantum dots

\section{Introduction}

In a two-dimensional bilayer system of charged particles where the separation of the layers are large enough to prevent tunnelling, an external current applied in one (active) layer can drag the charges in the adjacent (passive) via the inter-layer Coulomb interaction, and thus, induce a current. This phenomenon, known as the Coulomb Drag (CD) effect, which was proposed theoretically [1], and investigated experimentally [2]. The $\mathrm{CD}$ is of fundamental interest, since it can be utilized as a probing tool to investigate the screened interaction in mesoscopic transport systems. A quantitative measure of the $\mathrm{CD}$ is the transresistivity $\rho_{21}=E_{2} / J_{1}$ where $J_{1}$ is the applied current in the active layer and $E_{2}$ is the induced electric field in the passive layer.

\footnotetext{
*Corresponding author. Tel.: + 498921804532.

E-mail address: siddiki@fkf.mpg.de (A. Siddiki).
}

The presence of perpendicularly applied magnetic field enriches the physics of the $\mathrm{CD}$ effect by introducing Landau levels. The electrostatic and transport properties of the bilayer system were investigated first within the independent electron picture [3] and recently within a self-consistent screening scheme [4,5]. The main outcomes of the self-consistent scheme are (i) the formation of incompressible strips in the active layer at integer Landau filling factor values, which affects the density and potential profile in the passive layer and (ii) the tilting of the total potential within the active layer in the out-oflinear response (OLR) regime, induced by a large external current [6].

In this work, we combine these effects to formulate the interaction between the active and passive layers within the OLR regime, in order to provide a self-consistent description of the $\mathrm{CD}$ effect. We investigate the effect of the current intensity, temperature and density mismatch between the layers on the inter-layer Coulomb interaction in the OLR regime. 


\section{Model, results and discussion}

The electron bilayer system in the present work consists of two GaAs quantum wells (having a width of $2 d \sim 3 \mu \mathrm{m}$ ) that are sandwiched between two silicon doped thick (AlGa)As layers. This structure is grown on top of a GaAs substrate. The layers are translationally invariant. The electron density of the top layer is manipulated by a top gate. The electron layers are symmetric (with respect to the growth direction) and $(h \sim) 10-30 \mathrm{~nm}$ apart separated by an undoped (AlGa)As layer. Such a bilayer system is known to be electronically decoupled and can be represented by two different electrochemical potentials. We note that the electron tunnelling between the layers is not possible. Both of the electron channels are formed in the interval $-b<x<b$, where $|d-b|$ sets the depletion length.

The total electrostatic potential of an electron on the line $(x, z)$ due to a line-charge at $\left(x_{0}, z_{0}\right)$ is given by [5]

$V(x, z)=V_{b g}(x, z)+V_{\mathrm{H}}(x, z)$

and

$V_{\mathrm{H}}(x, z)=\frac{2 e^{2}}{\bar{\kappa}} \int_{x_{l}}^{x_{r}} \mathrm{~d} x_{0} K\left(x, x_{0}, z, z_{0}\right) n_{\mathrm{el}}\left(x_{0}, z_{0}\right)$,

where $-e$ is the charge of an electron, $\bar{\kappa}$ an average background dielectric constant, and the kernel $K\left(x, x_{0}, z, z_{0}\right)$ solves Poisson's equation under the relevant boundary conditions given by

$K\left(x, x_{0}, z, z_{0}\right)=-\ln \left(\frac{\cos ^{2} \frac{\pi}{4 d}\left(x+x_{0}\right)+\gamma^{2}}{\sin ^{2} \frac{\pi}{4 d}\left(x-x_{0}\right)+\gamma^{2}}\right)$,

where the $z$-dependence is given by $\gamma=\sinh \left(\pi\left|z-z_{0}\right| / 4 d\right)$. The confining (background) potential is obtained by inserting a constant number density $\left(n_{0}\right)$ of the background charges into Eq. (2). The gate can be described by an induced charge distribution $n_{\mathrm{g}}(x)$, that is residing on the surface. In order to obtain a flat (gate) potential profile in the bulk we choose the induced charge distribution as $n_{\mathrm{g}}(x)=n_{\mathrm{g}}^{0}\left(1+\alpha(x / d)^{2}\right)$ where $n_{\mathrm{g}}^{0}$ determines the strength of the gate potential, whereas $\alpha(=0.7)$ gives the slope of the induced charge distribution. With such treatment of the gate, the average electron densities can be modified while keeping the depletion length constant. The Hartree potential can be calculated from Eq. (2) for the top (bottom) layer yielding a total potential

$$
\begin{aligned}
V(x, z)= & -V_{b g}^{\mathrm{T}}(x, z)-V_{b g}^{\mathrm{B}}(x, z)-V_{g}(x, z) \\
& +V_{\mathrm{H}}^{\mathrm{T}}(x, z)+V_{\mathrm{H}}^{\mathrm{B}}(x, z) .
\end{aligned}
$$

The electron densities are calculated within the TFA:

$n_{\mathrm{el}}^{\mathrm{T}, \mathrm{B}}(x)=\int \mathrm{d} E D(E) f\left(\left[E+V\left(x, z_{\mathrm{T}, \mathrm{B}}\right)-\mu_{\mathrm{T}, \mathrm{B}}^{*}\right]\right)$,

with $D(E)$ is the Landau density of states (DOS), $f(E)=$ $1 /\left[\exp \left(E / k_{\mathrm{B}} T\right)+1\right]$ is the Fermi function, $\mu_{\mathrm{T}, \mathrm{B}}^{*}$ is the chemical potential (being constant in the equilibrium state) and $z_{\mathrm{T}, \mathrm{B}}$ is the position of top (bottom) layer, respectively. We employ the Gaussian broadened Landau DOS [6]. The self-consistent numerical calculations start with obtaining the confining potential created by its own donors for each layer and the electron densities and the intra-layer and inter-layer Hartree potentials [7] at zero temperature and zero magnetic field. The energies are scaled by the average pinch-off energy $E_{0}=2 \pi e^{2} n_{0} d / \bar{\kappa}$. The lengths are scaled by the screening length $a_{0}\left(=a_{\mathrm{B}}^{*} / 2\right)$ expressed in terms of effective Bohr radius, $a_{\mathrm{B}}^{*}=\bar{\kappa} \hbar^{2} /\left(m e^{2}\right)$. The electron density and the electrostatic potential for finite temperature and magnetic field can be calculated self-consistently by the above scheme within the TFA.

The crucial and distinguishing feature of the approach we implement is that we neither rely on a one-electron description nor use any phenomenological parameters to account for electron interactions, both for inter- and intralayer. In the next step, we drive a relatively high current from the active (here, top) layer and calculate the effect of this on the passive layer. We use a local version of Ohm's law to describe current densities employing a Gaussian broadened DOS defining the conductivity model $[6,8]$. We assume a local thermal equilibrium, where local quantities vary slowly on the quantum mechanical length scales. In the presence of current, it is well known that the Landau levels are tilted due to the Hall potential developed [6], therefore the electrochemical potential $\mu_{\mathrm{T}, \mathrm{B}}^{*}$, becomes position dependent and thus must be included within the self-consistent scheme. This brings two other self-consistent loops: (i) the re-calculation of the $\mu_{\mathrm{T}, \mathrm{B}}^{*}(x)$ in each layer; (ii) the response of the other layer to this modification. In this work, we also include this tilting to investigate its effect on

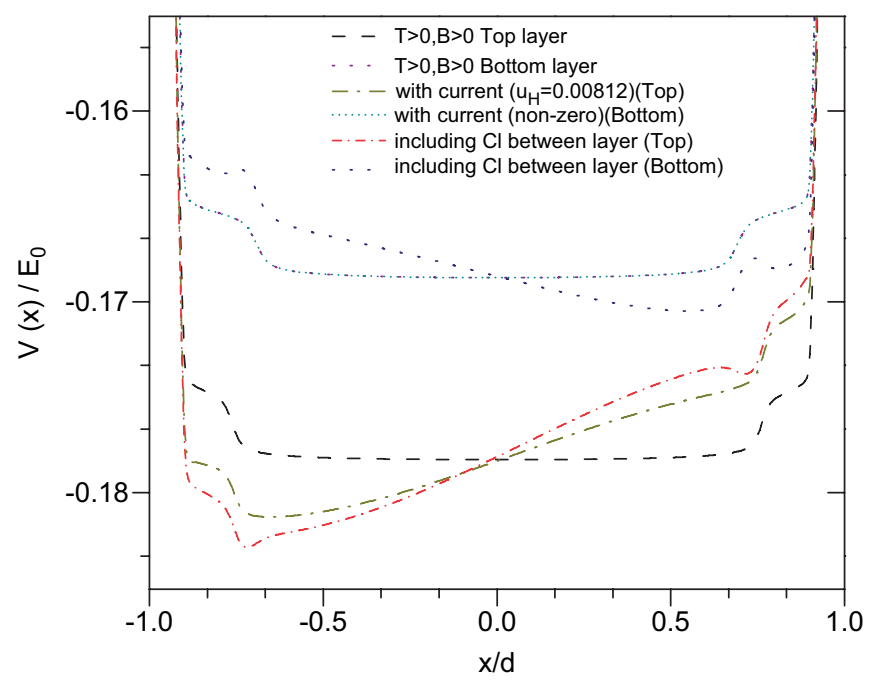

Fig. 1. The calculated potential profiles as a function of lateral coordinate, for zero and finite current applied to the top layer. The gate potential is chosen such that the resulting density mismatch is at the order of few percents, namely $V_{g} / E_{0}=0.05$. Calculations are done at $k_{\mathrm{B}} T / E_{0}=5.5 \times 10^{-3}$ for $n_{0}=4 \times 10^{11} \mathrm{~cm}^{-2}$ and inter-layer spacing of $h=12 \mathrm{~nm}$. The $B$ field is selected such that $\hbar \omega_{c} / E_{0}=5.5 \times 10^{-4}$ assuming a fixed depletion length $b / d=0.9$ 
the potential (see Fig. 1) and the density profile of the passive layer.

Here, we aim to show the effect of the combination of the inter- and the intra-layer Coulomb interactions together with the effect of external current driven in at least one of the layers. If one drives a current perpendicular to the applied magnetic field a Hall potential develops in the transverse direction to the current, i.e. Hall potential, resulting from the Lorentz force. If the system is all compressible, the Hall potential is linear in $x$, as one would expect to observe in a normal metal. Here we observe a similar behavior for the top layer, depicted in Fig. 1. On the other hand, the response of the second layer is as expected in the first glance, since, if the top layer has a tilted electrochemical potential in one direction the other should be the opposite. In Fig. 1, we show the self-consistently calculated potential profiles of both layers. For zero bias the top layer (broken black line) and the bottom layer (dotted cyan line) are symmetric in $x$. However, for a large current intensity $\left(U_{H}\right.$, see Ref. [6]) at the top layer to induce an electrochemical potential of this layer (red short dashed-dotted lines) a tilt is induced at the bottom layer with an opposite slope (dark blue dotted lines). More interestingly, if a finite current is driven from the bottom layer with same current direction of the top layer, the tilting observed is slightly suppressed (brown dasheddotted line).

In summary, by exploiting the smooth variation of the external potential we have explicitly calculated the Cou- lomb interaction in and between the bilayer quantum Hall samples in the presence of a strong current driven in one layer. Our preliminary results indicate that, a strong Coulomb coupling induces modifications in the potential landscape of both layers, which clearly points that a selfconsistent microscopic model is necessary to grasp the underlying physics of the $\mathrm{CD}$ effect observed in the bilayer systems.

\section{Acknowledgments}

The authors acknowledge the support of the Marmaris Institute of Theoretical and Applied Physics (ITAP), TUBITAK Grant 105T110, SFB631 and DIP.

\section{References}

[1] P.J. Price, Physica B 117 (1983) 750.

[2] T.J. Gramila, J.P. Eisenstein, A.H. MacDonald, L.N. Pfeiffer, K.W. West, Phys. Rev. Lett. 66 (1991) 1216; J.P. Eisenstein, L.N. Pfeiffer, K.W. West, Bull. Amer. Phys. Soc. 42 (1997) 486

[3] A.P. Jauho, H. Smith, Phys. Rev. B 47 (1993) 4420;

M.C. Bonsager, K. Flensberg, B.Y.K. Hu, A.P. Jauho, Phys. Rev. B 56 (1997) 10314.

[4] A. Siddiki, S. Kraus, R.R. Gerhardts, Physica E 34 (2006) 136.

[5] A. Siddiki, Phys. Rev. B 75 (2007) 155311.

[6] K. Güven, R.R. Gerhardts, Phys. Rev. B 67 (2003) 115327.

[7] A. Siddiki, R.R. Gerhardts, Phys. Rev. B 68 (2003) 125315.

[8] A. Siddiki, R.R. Gerhardts, Phys. Rev. B 70 (2004) 195335. 